INPLASY

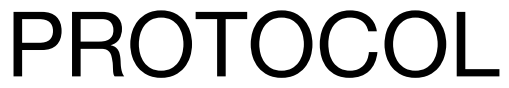

To cite: Fukuta et al. Influenza vaccination in patients with heart failure: a protocol for meta-analysis of randomized controlled trials. Inplasy protocol 202210115. doi: 10.37766/inplasy2022.1.0115

Received: 23 January 2022

Published: 23 January 2022

Corresponding author: Hidekatsu Fukuta

fukuta-h@med.nagoya-cu.ac.jp

Author Affiliation:

Nagoya City University Graduate School of Medical Sciences.

Support: The faculty research expenses.

Review Stage at time of this submission: The review has not yet started.

Conflicts of interest:

None declared.

\section{Influenza vaccination in patients with heart failure: a protocol for meta-analysis of randomized controlled trials}

\author{
Fukuta, $\mathrm{H}^{1}$; Hagiwara, $\mathrm{H}^{2}$; Kamiya, $\mathrm{T}^{4}$.
}

Review question / Objective: Heart failure is a major public health problem. Although there have been significant advances in the management of heart failure, the mortality and morbidity in heart failure patients remain high. Heart failure patients are susceptible to influenza-related complications including acute heart failure exacerbations and secondary infections such as pneumonia, both of which lead to significant morbidity and mortality. An earlier meta-analysis of observational cohort studies reported that influenza vaccination was associated with reduced risk of mortality in heart failure patients. Although there are no published randomized controlled trials (RCTs) on the effect of influenza vaccination on clinical outcomes in heart failure patients, there are several on-going RCTs examining the effect in these patients. We aim to conduct a meta-analysis of RCTs to assess the efficacy and safety of influenza vaccination in heart failure patients.

Information sources: The electronic databases for literature search will include PubMed, Scopus, Cochrane Library, and Web of Science.

INPLASY registration number: This protocol was registered with the International Platform of Registered Systematic Review and Meta-Analysis Protocols (INPLASY) on 23 January 2022 and was last updated on 23 January 2022 (registration number INPLASY202210115).

\section{INTRODUCTION}

Review question / Objective: Heart failure is a major public health problem. Although there have been significant advances in the management of heart failure, the mortality and morbidity in heart failure patients remain high. Heart failure patients are susceptible to influenza-related complications including acute heart failure 
exacerbations and secondary infections such as pneumonia, both of which lead to significant morbidity and mortality. An earlier meta-analysis of observational cohort studies reported that influenza vaccination was associated with reduced risk of mortality in heart failure patients. Although there are no published randomized controlled trials (RCTs) on the effect of influenza vaccination on clinical outcomes in heart failure patients, there are several on-going RCTs examining the effect in these patients. We aim to conduct a meta-analysis of RCTs to assess the efficacy and safety of influenza vaccination in heart failure patients.

Condition being studied: Patients with chronic heart failure.

\section{METHODS}

Search strategy: The electronic databases for literature search will include PubMed, Scopus, Cochrane Library, and Web of Science. For search of the eligible studies, the following key words and Medical Subject Heading will be used: heart failure, influenza, vaccine, randomized. Only articles published in the English language will be included.

Participant or population: Patients with heart failure.

Intervention: Influenza vaccination.

Comparator: Usual medical therapy or placebo control group.

Study designs to be included: Randomized controlled trials.

Eligibility criteria: Studies will be considered eligible if they; (1) included heart failure patients; (2) were randomized controlled trials ; (3) used influenza vaccine; and (4) compared with usual medical therapy or placebo control group.

Information sources: The electronic databases for literature search will include PubMed, Scopus, Cochrane Library, and Web of Science.
Main outcome(s): The primary outcome of interest will be cardiovascular death.

Additional outcome(s): The secondary outcomes of interest will be all-cause death, non-fatal myocardial infarction, nonfatal stroke, hospitalization for heart failure, and hospitalization for any cause.

Quality assessment / Risk of bias analysis: The Cochrane Risk of Bias tool will be used to assess the quality of included randomized controlled trials. The quality of evidence for the outcomes will be evaluated by use of the Grading of Recommendations Assessment, Development and Evaluation system. The quality of evidence will be evaluated across the domains of risk of bias, consistency, directness, precision, and publication bias.

Strategy of data synthesis: For each outcome, the pooled estimate of hazard ratio and $95 \% \mathrm{Cl}$ will be calculated with a fixed-effects model. The heterogeneity will be assessed using the Cochran's $Q$ chisquare test and 12 statistic; for the Cochran's $Q$ chi-square test and 12 statistic, a $p$ value of $<0.1$ and $I 2>50 \%$, will be considered significant, respectively \{21474\}. When there is significant heterogeneity, the data will be pooled using a random-effects model. A two-tailed $\mathbf{P}<$ 0.05 will be considered statistically significant. Publication bias will be assessed graphically using a funnel plot and mathematically using Egger test.

Subgroup analysis: Meta-analysis will be performed separately for patients with reduced ejection fraction and those with preserved ejection fraction.

Sensitivity analysis: Meta-regression will be used to determine whether the effect of influenza vaccination is confounded by baseline clinical characteristics.

Country(ies) involved: Japan.

Keywords: influenza; vaccine; heart failure; meta-analysis. 
Contributions of each author:

Author 1 - Hidekatsu Fukuta.

Email: fukuta-h@med.nagoya-cu.ac.jp

Author 2 - Hiromi Hagiwara.

Email: crhiroha@med.nagoya-cu.ac.jp

Author 3 - Takeshi Kamiya.

Email: kamitake@med.nagoya-cu.ac.jp 